\title{
El consumo de alcohol durante el tratamiento y seguimiento con drogodependientes ${ }^{1}$
}

\author{
Manuel Sanabria Carretero² \\ Santiago de Compostela
}

\begin{abstract}
Se estudia la influencia del consumo de alcohol en la recuperación de drogadictos sometidos a tratamiento. Se relacionan los niveles de consumo de drogas ilegales y de alcohol de antes, durante y después del tratamiento en una muestra de 82 pacientes seguidos durante un año y medio. Se evidencia que tras la intervención aumenta el consumo de alcohol en el $50 \%$ de adictos y llega a niveles excesivos en un 10-13\%. El mayor consumo coincide con la finalización del tratamiento. Posteriormente ( 18 meses) baja a niveles próximos a los iniciales. Estos resultados no permiten interpretar dicho aumento mediante la hipótesis de la sustitución, es decir, el desvío del consumo hacia sustancias más toleradas socialmente.
\end{abstract}

Palabras clave: alcohol, drogadicto, tratamiento, seguimiento, sustitución.

\section{Alcohol use by drug addicts during the treatment and follow-up}

This study is about the use of alcohol and its influence during the recuperation of drug addict patients undergoing treatment. Levels of the use of illegal drugs and alcohol were compared before, during and after the treatment of 82 patients assessed over a one-and-a-half year follow-up study. The results show that after the intervention there is an increase of alcohol use by $50 \%$ of the addicts. The $10-13 \%$ exhibit excessive levels. The higher alcohol use coincide with the end of the treatment. This tendency changes later ( 18 months) into similar levels as the initial ones. These results do not allow to consider the aforementioned increase as a confirmation of the substitution hypothesis, that is the alcohol use-redirection towards other more socially tolerated substances.

Key words: alcohol, drug addict, treatment, follow-up, substitution.

I El presente trabajo se ha llevado a cabo en cinco Centros Asistenciales de la Red Gallega de Drogodependencias. La coordinación del mismo corrió a cargo del Departamento de Psicología Clínica y Psicobiología de la Universidad de Santiago y formó parte de un proyecto de investigación más amplio.

2 Doctor en psicología por la Universidad de Santiago de Compostela. Su área de especialización e investigación es la drogodependencia. Ha escrito numerosos artículos sobre ese tema. Ha trabajado en el Programa de Rehabilitación de Drogodependientes Proyecto Hombre, con el que continúa colaborando. Ha desarrollado el Programa de Prevención del Consumo de Drogas CINENSINO. Actualmente trabaja como profesor y psicólogo. C/ Rosalía de Castro, 55 - $4^{\circ}$ C - 15895 Milladoiro - Santiago de Compostela - Coruña España. Correo electrónico: masanabria@teleline.es. 

En el campo terapéutico de las drogodependencias es evidente que el paso del paciente por un programa de tratamiento se asocia cambios en sus hábitos de consumo. Si bien la abstinencia total es el objetivo deseable para todos los pacientes, la realidad nos dice que para muchos resulta inalcanzable, operándose otro tipo de cambios que van desde la reducción del consumo hasta el desvío hacia otras sustancias.

En los estudios epidemiológicos con pacientes adictos el consumo de alcohol suele ser mayoritario, llegando a tener una relevante presencia en la cesta del policonsumo. La mitad de los adictos opiáceos son consumidores habituales de alcohol y más del $25 \%$ de los drogadictos sometidos a tratamiento podrían ser etiquetados indistintamente como alcohólicos o heroinómanos (Kreer, 1990; Rounsaville, Weisman, Kleber y Wilber, 1983; Weller, Halikas y Darwish, 1980; Westrin, 1990). Así pues, dada la situación, resulta de interés estudiar la evolución de los pacientes adictos en cuanto al consumo de alcohol a lo largo del tratamiento y seguimiento. En este sentido, suelen observarse cambios tendentes a aumentar la ingesta de alcohol a lo largo del proceso terapéutico. Stimson y Oppenheimer (1982) señalan que algunos adictos opiáceos permanecen poco tiempo abstinentes tras la implicación en un tratamiento, iniciándose en el consumo de sustancias como el alcohol u otras.

Así pues, cabe sospechar que si después del tratamiento se incrementa el consumo de alcohol, dicho ascenso pudiera reunir características compensatorias, lo que ha dado pie para aventurar la hipótesis de la sustitución, que coinciden con lo señalado en bastantes investigaciones que relacionan la reducción del consumo de drogas con aumentos en el consumo de alcohol (Arias, López-Ibor y Ochoa, 1997; Berglund et al., 1991; Lehman, Barret y Simpson, 1990; Sán- 
chez-Carbonell, Brigós y Camí, 1989; Simpson, Joe, Lehman y Sells, 1986; Simpson y Lloyd, 1981; Simpson y Sells, 1983; Stimson y Oppenheimer, 1982; Uchtenhagen y Zimmer, 1987).

En algunos casos se ha llegado a observar incrementos en el consumo semanal de alcohol de hasta el 65\% (Sheffet, Quinones, Doyle, Lavenhar, Nakah y Louria, 1980). Igualmente, Hubbard, Rachal, Craddock y Cavanaugh (1984) señalan que tres de cada diez pacientes pueden ser considerados como bebedores duros, dado que el tratamiento no parece incidir en la reducción del consumo de alcohol. El trabajo de Lehman, Barrett y Simpson (1990) aclara algunos de estos aspectos. Realizaron un estudio con 298 pacientes adictos y encontraron un grupo cuyo consumo alcohólico pudiera tener connotaciones de sustitución. Se diferenció dentro de éste dos subgrupos, uno con alto consumo y el otro con bajo. Sin embargo, el historial alcohólico diferenciaba a los posibles sujetos de sustitución de los de no sustitución, con un mayor consumo previo los primeros. La cuestión de la sustitución se volvería más confusa, ya que pudiera tratarse de la reanudación del patrón de consumo previo al tratamiento que, a su vez, había sido interrumpido o solapado por la propia adicción.

Para otros, el incremento del consumo alcohólico tras el tratamiento tendría una función instrumental, que se relaciona con problemas de ansiedad o de estrés. Los pacientes logran enfrentar estados de ánimo negativos ayudados por el uso de esta sustancia (Flykholm, 1979; Kosten, Rounsaville y Kleber, 1987).

\section{El presente estudio}

Con el propósito de clarificar algunos de los aspectos expuestos, en el presente estudio nos proponemos valorar el papel que desempeña la ingesta de alcohol en pacientes adictos sometidos a tratamiento, así como su significado e implicaciones, tanto para el tratamiento como para el seguimiento. 
El consumo de alcohol durante el tratamiento y seguimiento con drogodependientes

\section{Variables}

Las variables criterio que hemos utilizado son las que hacen referencia al consumo/abstinencia de las diferentes sustancias. Se distingue entre la heroína, por ser la sustancia por la que mayoritariamente se solicitó tratamiento y el resto de sustancias u "otras drogas", utilizando como criterio la frecuencia del consumo en el último mes, pudiendo oscilar entre cero (ningún consumo) y ocho (más de un consumo diario).

Para el caso del alcohol la variable está expresada en los centímetros cúbicos de alcohol puro consumidos al mes, calculados mediante una tabla de conversión.

El Indice Global de Adaptación (IGA) resume en una puntuación que oscila entre 0 y 4 la situación evolutivo-terapéutica del paciente. Al paciente se le suma un punto por cada una de las condiciones establecidas que cumpla, a saber: no consumir heroína, no consumir otras drogas; en tercer lugar, trabajar o estar matriculado en algún curso y, por último, no haber cometido actividad ilegal en los últimos 3 meses.

\section{Metodología}

Teniendo en cuenta las interrelaciones que el consumo de alcohol puede tener en la evolución terapéutica de los pacientes adictos, nos proponemos estudiar y valorar algunas de sus implicaciones. A tal fin hemos adoptado un diseño longitudinal de panel, en el que se ha seguido a la muestra seleccionada durante un año y medio, y se ha llevado a cabo valoraciones de los sujetos en intervalos de 6 meses.

\section{Participantes}

La muestra total utilizada para el estudio la forman 82 personas adictas que acudieron a tratamiento por problemas de consumo de 
sustancias ilegales en 5 centros de la Red Asistencial de Drogodependencias de la Comunidad Autónoma de Galicia. La edad media de estos pacientes se sitúa en los 25 años.

En el momento de acudir a tratamiento, la totalidad de la muestra es consumidora habitual de diversas sustancias, siendo el policonsumo el perfil más característico. La sustancia más consumida es la heroína, ya que el $72 \%$ de la muestra lo hace diariamente, seguida del hachís, tranquilizantes y cocaína consumidas de manera semanal por el $53 \%, 47 \%$ y $41 \%$, respectivamente.

Los participantes eran asignados a diferentes programas (Libre de Drogas, Mantenimiento con Metadona) teniendo en cuenta sus características. La duración media del tratamiento oscilaba entre los 6 meses y el año, como término medio.

\section{Procedimiento}

Una vez que se contactó con las distintas Unidades Asistenciales de Drogodependencias de la comunidad gallega y se les explicó el plan de trabajo, se elaboró un perfil prototipo del paciente en función de variables de carácter sociodemográfico. Teniendo en cuenta los usuarios atendidos el año anterior, se fijó un número proporcional y representativo de pacientes a reclutar para el estudio.

Los pacientes fueron seleccionados a medida que acudían a los centros, siempre y cuando encajaran en el perfil establecido. Se solicitaba su consentimiento para ser incluidos en el estudio y evaluarlos en diferentes momentos. Los contactos con los pacientes se realizaban a través del propio centro. Cuando esta alternativa no era posible se determinaba con el paciente otro lugar alternativo (cafeterías, bibliotecas...) para poder realizar las entrevistas. En algunas ocasiones, ante la imposibilidad de contar con la presencia física del paciente, la entrevista se realizó por teléfono. Los entrevistadores eran licenciados en psicología y asistentes sociales a los que se les entrenó 
previamente; además, tuvieron un rodaje de una semana para familiarizarse con los cuestionarios.

Los tratamientos.- Los pacientes fueron asignados fundamentalmente a dos tipos de tratamiento: Programas Libres de Drogas y de Mantenimiento con Metadona. Dentro de los primeros había dos tipos de modalidades, una de carácter Ambulatorio, donde el paciente acude al centro a las sesiones de terapia y apoyo (generalmente gran parte de la mañana) y pasa el resto de día en compañía de la familia o persona responsable; por otro lado, el Centro de Día se caracteriza por una mayor estancia del paciente en el centro, donde se compaginan las actividades terapéuticas con otras complementarias de carácter ocupacional, formativo y de ocio.

En los tratamientos de Mantenimiento con Metadona, la intervención gira en torno al uso de esa sustancia con fines terapéuticos: el paciente acude al centro a recibir su dosis y terapia de apoyo, mientras se trata de involucrarlo en otro tipo de actividades con la intencionalidad de que pueda acceder a programas Libres de Drogas.

Así pues, teniendo en cuenta que muchos pacientes fueron alternando de modalidad terapéutica (de Mantenimiento de Drogas a Libres de Drogas y viceversa) y en función de su evolución, no vamos a analizar los resultados según el tipo de tratamiento por el solapamiento que existe en nuestra muestra, sino que vamos a considerar el evento terapéutico como criterio único con independencia de la modalidad.

\section{Instrumentos}

Se elaboró la Entrevista Estructurada de Recogida de Datos (EERD). Es una adaptación, con algunas modificaciones, de la versión utilizada en el proyecto EMETYS (Sánchez-Carbonell, Brigós y Camí, 1989), la cual a su vez se basó en el ASI (Addiction Severity Index) (Mclellan, Luborsky, Cacciola, Griffith, Evans, Barr y O’Brien, 1985). 
El instrumento explora de manera progresiva lo relativo al área de consumo de drogas. Se va precisando con preguntas que tienden a medir la amplitud o duración del consumo y la intensidad del mismo desde el pasado hasta los 30 últimos días, considerando en este caso, la referencia del consumo actual. Además, y para el caso del alcohol, se incluyó una tabla de conversión en la que se especifican las equivalencias de las unidades consumidas en relación con los centímetros cúbicos (c.c:) de alcohol puro contenidos.

\section{Resultados}

Para apreciar el comportamiento de los sujetos en cuanto a la ingesta de alcohol comparamos la evolución del consumo desde antes del tratamiento y a lo largo del seguimiento. A primera vista, tal como se puede apreciar en la Figura 1, los niveles de alcohol tienden a aumentar una vez que los pacientes ingresan en el tratamiento. El aumento alcanza los niveles más altos a los 12 meses de iniciar el tratamiento y luego disminuye ligeramente a los 18 meses.

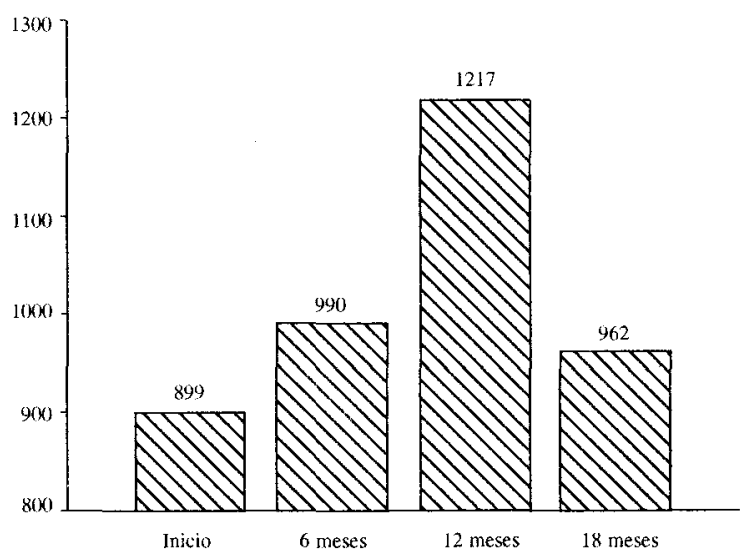

Figura 1. Evolución del consumo de alcohol. 
Si se analizan los cambios en los niveles de consumo entre todos los pases y los comparamos mediante un análisis de varianza de medidas repetidas, se aprecian diferencias significativas $\left(F_{1.81}=66.66 ; p\right.$ $=.00$ ) entre los cuatro periodos, siendo más llamativo el aumento experimentado a los 12 meses. Parece, por tanto, que este intervalo temporal supone mayor riesgo de consumo, lo que coincide con la finalización del tratamiento. Esto implica una mayor capacidad para gestionar su tiempo al margen de la tutela terapéutica.

Al mismo tiempo la incidencia de esta variable parece simular un efecto rebote, ya que tras la implicación del paciente en el programa, el consumo de alcohol tiende a aumentar. Más adelante desciende (18 meses) hasta niveles ya más próximos a los de antes de iniciar el tratamiento.

Con el fin de profundizar en estas cuestiones, se seleccionó los sujetos que habían aumentado su consumo de alcohol con relación al pase inicial. El Cuadro 1 recoge estos resultados. Se observa que la mitad de la muestra aumenta su ingesta etílica con relación al pase inicial. Si bien el número de pacientes que incrementa su consumo entre los 6 y 12 meses prácticamente no aumenta, sí aumenta la cantidad consumida. Ésta alcanza una media de 1217 c.c. de alcohol puro al mes.

\section{Cuadro 1}

Aumento del consumo de alcohol respecto al pase inicial $(N=82)$

\begin{tabular}{|ccccc|}
\hline $\begin{array}{c}\text { Tiempo } \\
\text { (meses) }\end{array}$ & \multicolumn{2}{c|}{ Aumento del consumo } & \multicolumn{2}{c|}{$\begin{array}{c}\text { Consumo }>75 \mathrm{cc} . / \text { día } \\
n\end{array}$} \\
\hline & $n$ & $\%$ & 13 & 15.8 \\
6 & 44 & 53.6 & 10 & 12.1 \\
12 & 45 & 54.8 & 9 & 10.9 \\
18 & 39 & 47.5 & & \\
\hline
\end{tabular}

Así pues, este efecto rebote no parece estar provocado por un incremento del número de pacientes consumidores de alcohol, sino más 
bien por un aumento de las cantidades consumidas por los mismos pacientes durante esa fase. Ahora bien, dentro del grupo que incrementa el consumo de alcohol, destaca un subgrupo formado por el 10-13\% de los pacientes para los que el consumo de alcohol sobrepasa los 75 c.c. de alcohol puro/día, lo que se considera un consumo excesivo. Este consumo pudiera revestir un carácter compensatorio, pues se desvía el hábito consumista hacia esta sustancia legal; sin embargo, tampoco podemos descartar que se trate de la recuperación de un nivel de consumo previo a la adicción, mermado o solapado por el consumo de otras drogas, aspecto en el que no podemos profundizar puesto que el historial de consumo no abarca suficientemente ese periodo.

Estos detalles sugieren que los resultados de los tratamientos respecto al consumo de algunas sustancias pueden ser efímeros e inestables justo después de la finalización de los mismos, debiendo hacerse valoraciones a medio plazo cuando el efecto rebote ya se haya estabilizado.

Posteriormente, con el fin de verificar si hay alguna relación entre el consumo de alcohol y el de otras drogas, relacionamos ambos tipos de consumo mediante un análisis de correlación de Pearson. Los únicos valores significativos son entre el cannabis (hachís) y alcohol, cuyos consumos tienden a aumentar en ambos casos (Cuadro 2). En el resto de sustancias ilegales no se aprecia ningún tipo de relación significativa.

\section{Cuadro 2}

Relación entre consumo de alcohol y Cannabis

\begin{tabular}{|ccc|}
\hline 6 meses & 12 meses & 18 meses \\
\hline$r=.17$ & $r=.34$ & $r=$ \\
$p=.04$ & $p=.00$ & $p=.03$ \\
\hline
\end{tabular}


Así pues, estos datos no parecen ajustarse al supuesto papel compensatorio atribuido al alcohol respecto a otras drogas, ya que la supuesta relación debería ser de tipo excluyente. Para el caso del hachís $y$ del alcohol ambos consumos se rigen por pautas complementarias. Para el resto de sustancias ilegales no se aprecian relaciones significativas en ninguná dirección.

Otra cuestión es tratar de averiguar si las pautas de ingesta alcohólica tras el tratamiento logran diferenciar a los pacientes que tienden a potenciar el consumo etílico de los que no lo hacen. En este sentido tampoco apreciamos diferencias entre ambos grupos en cuanto al nivel de adaptación final (IGA), ya que la $t$ de Student para muestras independientes no alcanza valor significativo. Entonces, el incremento del consumo de alcohol no estaría asociado a resultados más pobres para esos pacientes, dicho aumento no parece provocar un "efecto dominó" sobre otras sustancias ilegales.

A tenor de estos resultados parece más lógico pensar que el incremento del consumo alcohólico cumple una función instrumental que ayuda al paciente a hacer frente a situaciones estresantes o para demostrarse a sí mismo la capacidad de manejo o control sobre esta sustancia en contextos de interrelación o de ocio, como ya apuntaron Kosten, Rounsaville y Kleber (1987).

\section{Discusión}

Es evidente que la relación de los pacientes adictos respecto de algunas sustancias de consumo a lo largo de su proceso de recuperación sufre importantes cambios que conviene conocer y valorar en su justa medida para hacer más comprensible ese proceso y adaptarlo a las características de los pacientes. En esta línea, los datos de nuestro estudio indican que, tras la implicación del paciente adicto en un programa de tratamiento, se produce un aumento significativo en los 
niveles de ingesta alcohólica en relación con la línea base observada al iniciar el tratamiento. Estos datos son concordantes con los aportados en otras investigaciones y revelan que, al menos para la mitad de la muestra, se produce un aumento en los niveles de consumo de alcohol durante el tratamiento, mostrándose con más evidente en la etapa final del mismo (Arias, López-Ibor y Ochoa, 1997; Berglund et al., 1991; Labouvie y Pinsky, 2001; Oliveros, Caballero, Iruela y Baca, 1992).

El período de mayor riesgo se sitúa entorno a la finalización del tratamiento, hacia los 12 meses en nuestro caso, momento en el que la ingesta etílica se incrementa de forma significativa. Sin embargo, dicho incremento no se debe a la incorporación de nuevos pacientes que pudieran pasar de una situación de abstinencia a consumir, sino que el fenómeno lo protagonizan pacientes, concretamente un 54\% de la muestra, que desde el inicio del tratamiento han venido consumiendo en mayor o menor medida y que ahora incrementan su consumo de manera más notoria.

Este hecho, con frecuencia ya señalado en otras investigaciones (Arias, López-Ibor y Ochoa, 1997; Cuadrado, 1998; Stimson y Oppenheimer, 1982) parece apuntar a que el tratamiento es, en cierta medida, selectivo no incidiendo con igual eficacia en todo el conjunto de sustancias consumidas por los pacientes, sino que aquellas que gozan de mayor tolerancia y permisividad social, como es el caso del alcohol y hachís, parecen menos sensibles a los efectos del tratamiento. En cambio, otras sustancias, como los derivados opiáceos que constituyen la mayoría de las veces el objeto de la intervención, el tratamiento se asocia con una significativa disminución del consumo o la consecución de la abstinencia total.

Este fenómeno ha dado pie para sospechar que la ingesta etílica, durante y después del tratamiento, pudiera tener una función compensatoria, dando pie a la formulación de la hipótesis de la sustitu- 
ción por la frecuencia observada del fenómeno (Arias, López-Ibor y Ochoa, 1997; Berglund et al., 1991; Cuadrado, 1998; Lehman, Barret y Simpson, 1990). Lo cierto es que, para que se confirmen los supuestos de esta tendencia, la evolución del consumo de alcohol a lo largo del tratamiento y seguimiento debería guardar una relación inversa o excluyente respecto del consumo de otras sustancias ilegales, de manera que a medida que el individuo restringe el consumo de opiáceos deberían observarse incrementos en el consumo de alcohol. Sin embargo, los índices de correlación entre ambos tipos de variables ni indican relación ni es en la dirección esperada, de manera que esa supuesta relación no se da, al menos entre los pacientes de nuestra muestra.

Los resultados de nuestro estudio no parecen apoyar los supuestos de la hipótesis de la sustitución, más bien parecen desmarcarse de dicha hipótesis, de manera que el incremento de la ingesta alcohólica en la última fase del tratamiento tengan más que ver con la disminución de la tutela terapéutica y concesión de mayores cotas de autogestión del tiempo libre por parte del paciente. El incremento del consumo tendría una función de carácter instrumental, facilitador de interacción social en contextos de diversión como también destaca (Bullers, Cooper y Russell, 2001); aspecto que toma mayor relevancia para el sujeto a medida que se desliga del centro de tratamiento y busca una mayor integración en un medio sobre el que tenía ciertas restricciones por prescripción terapéutica.

Por otra parte y en contra de lo que pudiera pensarse, los sujetos que tienden a aumentar el consumo de alcohol durante la fase del tratamiento no van a alcanzar resultados más pobres en su evolución terapéutica final. Al comparar ambos grupos de sujetos, es decir, los consumidores de alcohol durante el tratamiento (la mitad de la muestra), con los que no lo hicieron no se aprecian diferencias significativas en cuanto a los resultados de la intervención. Así pues, igual que en el estudio de Cuadrado (1998), el consumo de alcohol durante la 
fase del tratamiento no parece predecir una peor evolución terapéutica con resultados más pobres.

Este hecho se ve corroborado en la etapa del seguimiento. A los 18 meses el efecto del consumo etílico alcista de la etapa anterior (12 meses) ha desaparecido, siendo equiparable a los niveles del pase de inicio, de manera que el incremento del consumo final responde a un patrón de transitoriedad, ya señalado también por otros autores, no siendo considerado como una pauta definitiva y permanente (Sánchez, Del Olmo, Morales y Tomás, 1998). Este hecho parece corroborar la función instrumental del consumo de alcohol en ese proceso de readaptación e integración al contexto social.

Una de las implicaciones terapéuticas a tener en cuenta y derivada de los resultados del presente estudio, tiene que ver con la conveniencia de realizar valoraciones por separado en el área toxicológica, considerando, por un lado, las sustancias de carácter legal y por otro las ilegales. Una valoración globalizadora, como se hace en algunas instituciones terapéuticas en las que se tiende a equiparar los consumos de alcohol a los de otras sustancias ilegales, puede ser un error. El incremento del consumo etílico no parece ser alarmista, tiende a remitir con el tiempo y no entorpece la evolución ni resultados del tratamiento.

Quizás sea aconsejable, por parte de los centros mostrarse más comprensivos y tolerantes con el consumo de alcohol, teniendo en cuenta que ese consumo alcista es, pasajero; de no hacerlo así corremos el riesgo de hacer valoraciones sesgadas de la evolución de los pacientes, en consecuencia, tomar decisiones erróneas, prolongar la permanencia en el tratamiento y desanimar a los pacientes con nuestros comentarios valorativos.

Otro aspecto importante y relacionado con el anterior, tiene que ver con el intervalo temporal tomado como referencia para estimar la 
eficacia de una intervención. Si dicho intervalo es corto y próximo a la finalización del tratamiento, podemos correr el riesgo de juzgar la eficacia de la intervención de manera desfavorable o poco eficaz; puesto que llama la atención que, justo al finalizar el tratamiento, la presencia del alcohol en la vida del sujeto sea más notoria. Dado que se trata de un fenómeno remitente, es aconsejable tomar como referencia un intervalo temporal más amplio valorando la situación evolutiva de los pacientes a medio o largo plazo cuando el posicionamiento del sujeto respecto al consumo de alcohol esté ya más consolidado y sea más realista.

Además, este hecho puede crear alarma en el entorno familiar del paciente y desacreditar la intervención por falta de eficacia, sería aconsejable informar a las familias de la posible evolución de los pacientes respecto al consumo de alcohol tras someterse a un proceso terapéutico por consumo de sustancias ilegales.

\section{Referencias}

Arias, F., López-Ibor, J. y Ochoa, E. (1997). Comorbilidad psiquiátrica en dependientes de opiáceos en tratamiento con naltrexona. Adicciones, 9 (2), 235-253.

Berglund, G., Bergmark, A., Björling, B., Grönbladh, L., Lindberg, S., Oscarsson, L., Olsson, B., Segraeus, V. y Stensmo, C. H. (1991). The SWEDATE Project: Interaction between treatment, client background and outcome in a one-year follow-up. Journal of Substance Abuse Treatment, 8, 161-169.

Buller, S., Cooper, M. L. y Russell, M. (2001). Social network drinking and adult alcohol involvement: a longitudinal exploration of the direction of influence. Addictive Behavior, 26, 181-199.

Cuadrado, P. (1998). Evolución de la dependencia alcohólica en tratamiento. Factores predictivos en un seguimiento de 5 a 7 años. Adicciones, 10, 335-341. 
Frykholm, B. (1979). Termination of the drug career. An interview study of the 58 ex-addicts. Acta Psychiatrica Scandinavica, 59, 370-380.

Hubbard, R., Rachal, J., Craddock, S. y Cavanaugh, E. (1984). Treatment outcome prospective study (TOPS): Client characteristics and behaviors before, during and after treatment. En F. Tims y J. Lidford (Eds.), Drug abuse treatment evaluation: Strategies, progress y prospects (NIDA Research Monograph, 51) (pp. 4268). Rockville, MD: US Department of Health and Human Services.

Kosten, T. R., Rounsaville, B.J. y Kleber, H. D. (1987). A 2.5 year follow-up of cocaine use among treated opioid addicts. Archives General of Psyciatry, 44(3), 281-284.

Kreer, M. J. (1990). Historical and medical aspects of methadone maintenance treatment. En C. Adamsson, B. Jansson, U. Rydberg y C. G. Westrin (Eds.), Evaluation of diferent programmes for treatment of drug addicts (pp. 61-76). Göteborg: Forskningsraadet.

Labouvie, E. y Pinskyi, I. (2001). Substance use and driving: the coexístanse of risky and safe behaviors. Addiction, 96, 473-484.

Lehman, W., Barrett, M. y Simpson, D. (1990). Alcohol use by heroin addicts 12 year after drug abuse treatment. Journal of Studies on Alcohol, 51, 3, 233-244.

McLellan, A. T., Luborsky, L., Cacciola, J., Griffith, B. A., Evans, F., Barr, H. L. y O’Brien, C. H. (1985). New data from the Addiction Severity Index. Reliability and validity in three centers. Journal Nervous and Mental Disease, 173, 7, 412-423.

Rounsaville, B. J., Weissman, M. M., Kleber, H. y Wilber, C. H. (1983). Heterogeneity of psychiatric diagnosis in treated opiate addicts. Archives General Psychiatry, 39, 161-166.

Sánchez, E., Del Río, R., Morales, E. y Tomás, V. (1998). Differences on variables in dependent subject to alcohol and the other illegal drugs. Drug and Alcohol Dependence, 52, 15-25.

Sánchez-Carbonell, J., Brigos, B. y Cami, J. (1989). Evolución de una muestra de heroinómanos dos años después del inicio del 
tratamiento: Proyecto EMETYST. Medicina Clínica, 92(4), 135139.

Sheffet, A., Quinones, M., Doyle, K., Lavenhar, M., Nakah, A. y Louria,

D. (1980). Assessment of treatment outcomes in drug abuse rehabilitation network: Newark, New Jersey. American Journal Drug and Alcohol Abuse, 7, 141-173.

Simpson, D., Joe, G., Lehman, W. y Sell, S. B. (1986). Addiction careers:

Etiology, treatment and 12 year follow-up outcomes. The Journal of Drug Issues, 16, (1) 107-121.

Simpson, D. y Lloyd, M. R. (1981). Alcohol use following treatment for drug addiction: a four year follow-up. Journal Study Alcohol, 42, 323-335.

Simpson, D. y Sells, S. B. (1983). Effectiveness of treatment for drug abuse: An overview of the DARP research program. Advances in Alcohol and Substance Abuse, 2, 7-29.

Stimson, G. y Oppenheimer, E. (Ed.) (1982). Heroin addiction. Treatment and control in Britain. Londres: Tavistock.

Uchtenhagen, A. y Zimmer, D. (1987). Psychosocial development following therapeutic and legal interventions in opiate dependence. A Swiss National Study. European Journal of Psychology of Education, 2(4), 443-458.

Weller, R. A., Halikas, J. y Darwish, H. (1980). Alcoholism in black male heroin addicts. British Joumal of Addictions, 75, 381-388.

Westrin, C. G. (1990). A health system perspective. En C. Adamsson, B. Jansson, U. Rydberg y C. G. Westrin (Eds.), Evaluation of diferent programmes for treatment of drug addicts. Göteborg: Forskningsraadet. 